\title{
PROPIEDADES DIELÉCTRICAS DE HIDRÓXIDOS DOBLES LAMINARES DE Mg:Al
}

\author{
Óscar Hernán Giraldo Osorio, ${ }^{\mathrm{a}, \mathrm{b}^{*}}$ Nayda Patricia Arias Duque ${ }^{\mathrm{a}, \mathrm{c}} \mathrm{y}$ \\ Jhon Mauricio Aguirre Cortés ${ }^{\mathrm{a}, \mathrm{c}}$
}

\begin{abstract}
RESUMEN
Este trabajo describe la síntesis y caracterización de dos hidróxidos dobles laminares $\mathrm{Mg}_{2} \mathrm{Al}-$ $\mathrm{NO}_{3}$ sintetizados mediante el método de coprecipitación. El comportamiento eléctrico de los materiales fue evaluado por espectroscopía dieléctrica. Los resultados del análisis de difracción de rayos X mostraron la formación de una estructura laminar; la presencia de todas las especies en la estructura del material sintetizado se confirmó por absorción atómica y espectroscopía de infrarrojo. Por microscopía electrónica de transmisión se observó agregados de partículas en forma de placas. Los resultados de espectroscopía dieléctrica obtenidos a temperatura ambiente muestran un comportamiento de polarización de orientación que sugiere la rotación de moléculas de agua a alta frecuencia y la rotación de aniones nitrato en el espacio interlaminar a baja frecuencia que puede ser responsable de la conductividad iónica observada en el material.

Palabras clave: Hidróxidos dobles laminares, propiedades dieléctricas, conductividad iónica.
\end{abstract}

\section{DIELECTRIC PROPERTIES OF Mg:AI LAYERED DOUBLE HYDROXIDES}

\begin{abstract}
This work describes the synthesis and characterization of $\mathrm{Mg}_{2} \mathrm{Al}-\mathrm{NO}_{3}$ layered double hydroxides nanomaterial prepared using a coprecipitation method. The electrical behavior of this material was evaluated using dielectric spectroscopy. The results from X-ray diffraction analysis showed the formation of lamellar structure, while the atomic absorption and infrared spectroscopy confirms the presence of all species inthe structure of the synthesized material. TEM shows clusters of plate-like particles and the dielectric spectroscopy results at room temperature show an orientational polarization behavior that suggests the rotation of water molecules at high frequency. TEM results also demonstrate the rotation of nitrate anions in the interlayer spacing at low frequency, which may be responsible for producing anionic conductivity.
\end{abstract}

Key words: Layered double hydroxides, dielectric properties, ionic conductivity.

\footnotetext{
a. Laboratorio de Materiales Nanoestructurados y Funcionales. Universidad Nacional de Colombia Sede Manizales.

b. Departamento de Física y Química. Facultad de Ciencias Exactas y Naturales. Universidad Nacional de Colombia Sede Manizales, Carrera 27 No. 64-60 Manizales, Caldas, Colombia.

c. Facultad de Ingeniería y Arquitectura. Universidad Nacional de Colombia Sede Manizales, Carrera 27

No. 64-60 Manizales, Caldas, Colombia

email: ohgiraldoo@unal.edu.co
} 


\section{INTRODUCCIÓN}

Los hidróxidos de dobles laminares (LDH), también conocidos como materiales tipo hidrotalcita o arcillas aniónicas, ${ }^{1-3}$ son estructuralmente similares al hidróxido de magnesio $\left[\mathrm{Mg}(\mathrm{OH})_{2}\right] .^{2}$ Los LDH se pueden sintetizar como fase cristalográfica pura bajo condiciones controladas a temperatura ambiente. ${ }^{3-5}$ Este tipo de materiales son considerados actualmente, como materiales nanoestructurados jerárquicos avanzados. ${ }^{4,5} \mathrm{Su}$ fórmula general puede representarse mediante $M_{1-x}^{2+} M_{x}^{3+}(\mathrm{OH})_{2}\left(A^{n-}\right)_{x / n} \cdot \mathrm{mH}_{2} \mathrm{O}$ donde $\mathrm{M}^{2+} \mathrm{y} \mathrm{M}^{3+}$ representan los cationes di y trivalentes, respectivamente; $\mathbf{m}$ representa el contenido de agua, $\mathbf{n}^{-}$la carga del anión y $\mathbf{x}$ el grado de sustitución del metal divalente. Los metales en esta estructura tienen coordinación octaédrica con los grupos $\mathrm{OH}^{-}$, en la que los octaedros comparten los bordes formando láminas ${ }^{3}$ de $4,8 \AA$ de espesor extendidas en dos dimensiones ${ }^{2}$. Las láminas están cargadas positivamente debido al grado de sustitución (x) del metal divalente por el trivalente; se ha reportado que x puede variar entre 0,2 a $0,4 .{ }^{5} \mathrm{El}$ material hospeda, a través de enlaces de hidrógeno e interacciones electrostáticas, iones negativos $\left(\mathbf{A}^{\mathrm{n}}\right)$ que compensan la carga positiva de la lámina. ${ }^{5} \mathrm{La}$ distancia entre las láminas cambia, dependiendo del anión huésped y puede variar entre $3,3 \AA$ a $4,9 \AA$ para $\mathrm{NO}_{3}^{-}$y $\mathrm{SO}_{4}^{2-}$, respectivamente; ${ }^{6}$ además, en la región interlaminar también se puede encontrar moléculas de agua que son representadas por $\mathbf{m}$ en la fórmula general del material. ${ }^{2,3}$ De esta manera, el material de dimensiones nano-métricas considerado como anfitrión puede tener diferentes propiedades, dependiendo de la especie huésped alojada en la región interlaminar. La distancia interlaminar se puede modificar mediante intercambio iónico con la incorporación de nanopartículas, moléculas orgánicas, polímeros, $\mathrm{ADN}$ y enzimas, entre otros sistemas. ${ }^{1-6} \mathrm{El}$ amplio rango de variaciones que se pueden realizar en los materiales tipo hidrotalcita permite importantes aplicaciones, como catalizadores, adsorbentes, sistemas para liberación controlada de medicamentos, sistemas para absorción de radiación UV, sensores, biocatalizadores, conductores iónicos en celdas combustibles y como electrodos en dispositivos de almacenamiento de energía. ${ }^{1-7}$ $\mathrm{Se}$ ha estudiado materiales LDH del tipo Ni-Al-Cl por espectroscopía de impedancia electroquímica, evaluando su comportamiento como función de la concentración del metal divalente, ${ }^{8}$ en el que la impedancia a bajas frecuencias fue dominada por la difusión de la especie interlaminar y el componente resistivo del transporte de masa decreció con el incremento en el estado de oxidación del níquel. Los LDH compuestos por Mg-Mn con carbonato interlaminar han mostrado propiedades de difusión. ${ }^{9}$ De igual forma, se ha estudiado las propiedades dieléctricas de los $\mathrm{LDH}$ de $\mathrm{Ca}-\mathrm{Fe}^{10}$ y Zn-Al conteniendo el anión nitrato y molibdato. ${ }^{11} \mathrm{Se}$ encuentran reportados pocos estudios sobre las propiedades dieléctricas de los $\mathrm{LDH}$ compuestos por $\mathrm{Mg}-\mathrm{Al}-\mathrm{NO}_{3}{ }^{12} \mathrm{El}$ propósito de este trabajo es proporcionar una aproximación fenomenológica al proceso que involucra el transporte de carga en HDL de $\mathrm{Mg}-\mathrm{Al}$ con ion nitrato como huésped.

\section{Procedimiento de síntesis}

\section{PARTE EXPERIMENTAL}

Se preparó un hidróxido doble laminar de $\mathrm{MgAl}$ con una relación molar $\mathrm{Mg} / \mathrm{Al}$ 2:1 por el método de coprecipitación a $\mathrm{pH}$ constante, descrito por Villegas y colaboradores. ${ }^{3}$ Para una síntesis típica se disolvieron 10,120 g de $\mathrm{Mg}\left(\mathrm{NO}_{3}\right)_{2} \cdot 6 \mathrm{H}_{2} \mathrm{O}$ (Merck, 99\%) en 20,0 $\mathrm{mL}$ de agua destilada desionizada (DDW) para preparar una solución 2,0M de la sal de nitrato del ion $\mathrm{M}^{2+}$. 
Por otro lado, se adicionó 7,340 g de $\mathrm{Al}\left(\mathrm{NO}_{3}\right)_{3} .9 \mathrm{H}_{2} \mathrm{O}$ (Merck, 99\%) en el mismo volumen de DDW para preparar una solución $1,0 \mathrm{M}$ de la sal de nitrato del ion $\mathrm{M}^{3+}$. La solución de las sales de nitratos se adicionó gota a gota a $20,0 \mathrm{~mL}$ de solución acuosa de $\mathrm{NaOH}$ (Carlo Erba, 98\%) preparada a $\mathrm{pH} 10,0 \pm 0,5$. El pH de la reacción se mantuvo en este rango con la adición de $\mathrm{NaOH} 2,0 \mathrm{M}$. El procedimiento se realizó con burbujeo constante de nitrógeno gaseoso para prevenir la introducción de iones carbonato provenientes del $\mathrm{CO}_{2}$ atmosférico.

\section{Caracterización}

La determinación de la cantidad total de $\mathrm{Mg}$ y Al se realizó por absorción atómica (AA) en un espectrómetro Perkin Elmer 3110. Los datos de difracción de rayos X en polvo (XRD) se obtuvieron en un difractómetro Rigaku Miniflex II, usando una fuente de $\mathrm{Cu} \mathrm{K} \alpha$ y un detector de NaI con una velocidad de barrido de $2 \%$ min, un tamaño de paso de $0,02{ }^{\circ}$ entre 3-70 ${ }^{\circ} 2$. Las micrografías se adquirieron en un Microscopio Electrónico de Transmisión (TEM) JEOL JEM-2010. El espectro infrarrojo con transformada de Fourier se analizó en un dispositivo 380 Nicolet, equipado con detector DTGS en el rango $4000-400 \mathrm{~cm}^{-1}$. Las mediciones dieléctricas AC de la muestra en polvo se realizaron en un equipo Solartron 1260 en un rango de frecuencias de $10 \mathrm{MHz}$ a $0,18 \mathrm{~Hz}$ a $100 \mathrm{mVrms}$ utilizando un portamuestras Solatron 12964A equipado con dos electrodos paralelos en configuración de dos puntas. La distancia entre el electrodo de trabajo y de referencia fue $1,57 \mathrm{~mm}$ con un diámetro efectivo del electrodo de trabajo de $20 \mathrm{~mm}$. El electrodo de trabajo se utilizó con un anillo de protección para evitar el efecto de líneas de corriente parásita en el borde de la muestra. Las condiciones ambientales para las mediciones dieléctricas fueron $21^{\circ} \mathrm{C}$ y $50 \%$ de humedad relativa. El circuito equivalente que ajusta los datos experimentales se simuló con el software Z-View software ${ }^{\circledR}$ (Scribner Associates).

\section{RESULTADOS Y DISCUSIÓN \\ Composición, estructura, morfología y espectro infrarrojo}

La relación molar $\mathrm{Mg} / \mathrm{Al}$ experimental determinada por A.A fue de 2,2, que está en buen acuerdo con el valor esperado de 2,0. El patrón de XRD (figura 1), evidenció que se obtuvo una fase pura de LDH. Su carácter laminar se confirmó por los picos armónicos a bajos valores de $2 \theta$. El espaciamiento basal, calculado en $8,9 \AA$ que corresponde al pico más intenso de difracción asociado a la difracción del plano (003), es consistente con la presencia de nitratos en la región interlaminar. ${ }^{2}$ Las distancias interplanares para los planos (006), (009), (012) y (110) fueron de $4,5 \AA, 2,6 \AA, 2,3 \AA$ y $1,5 \AA$, respectivamente; la representación esquemática de esta estructura se muestra en la figura 1a. El tamaño de cristal estimado mediante la ecuación de Debye- Scherrer fue de 5,0 nm. 


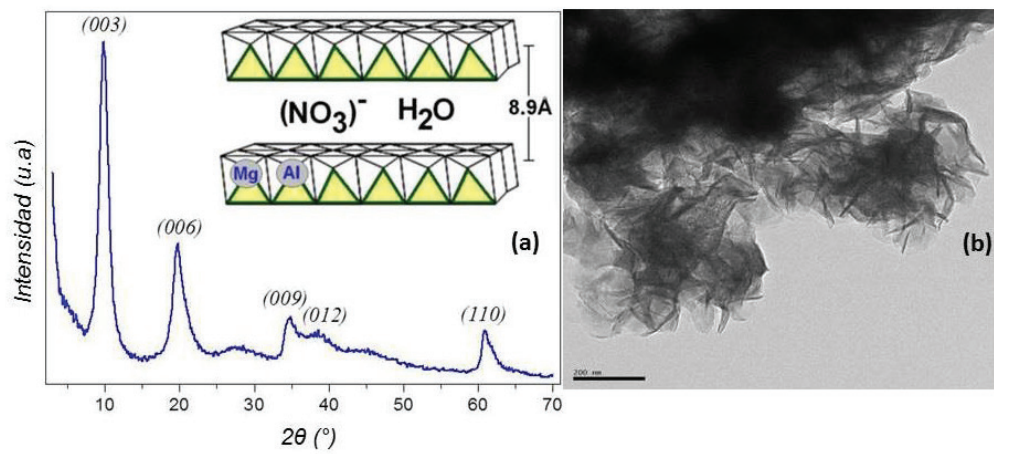

Figura 1. (a) Patrón de difracción de rayos X del LDH, Inserto: representación de la estructura laminar, (b) Microscopía electrónica de transmisión en la que se observa la conformación en placas de la estructura de los LDH.

La imagen de TEM para el material LDH Mg-Al (figura 1b) revela una morfología en placas característica de este tipo de estructuras ${ }^{3}$, que está compuesta por agregados de tamaño homogéneo. La figura 2 muestra el espectro vibracional FT-IR típico de este material. La banda centrada alrededor de $3489 \mathrm{~cm}^{-1}$ se asignó a las vibraciones de estiramiento de los grupos $\mathrm{OH}$ debido a las moléculas de agua localizadas en la región interlaminar y a los grupos $\mathrm{OH}^{-}$de la lámina. La vibración alrededor de $1635 \mathrm{~cm}^{-1}$ corresponde a las vibraciones de deformación de los grupos $\mathrm{OH}^{-}$de la estructura laminar y las bandas a $660 \mathrm{~cm}^{-1}$ y 558 $\mathrm{cm}^{-1}$ a las vibraciones metal-OH de la red cristalina de la estructura laminar. ${ }^{3}$ La presencia de aniones nitrato se confirmó en el espectro por la aparición de bandas vibracionales localizadas a 1370 y $820 \mathrm{~cm}^{-1}$ (modos $v 2$ y $v 3$ respectivamente). ${ }^{3}$

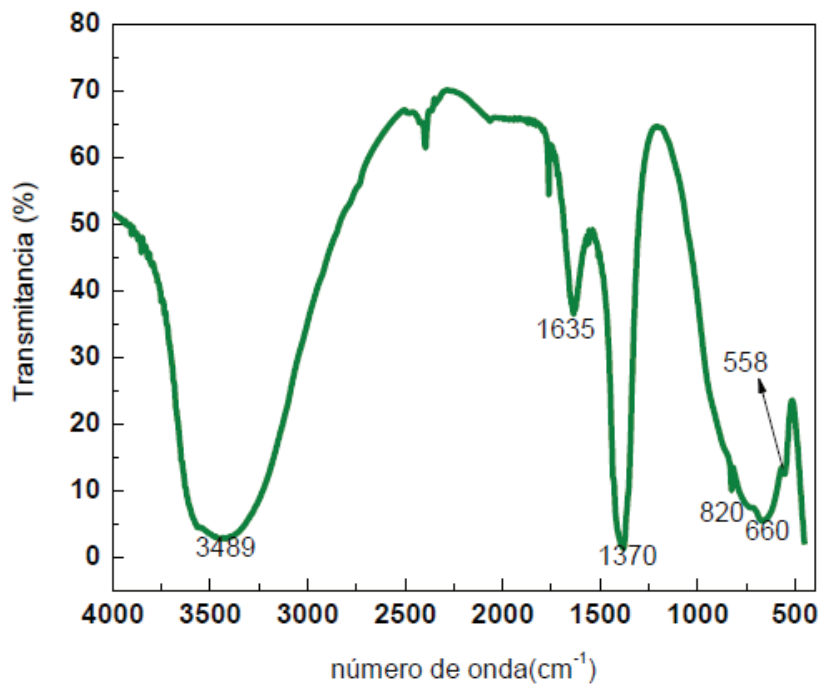

Figura 2. Espectro infrarrojo del hidróxido doble laminar $\mathrm{Mg}_{2} \mathrm{Al}_{-} \mathrm{NO}_{3}$. 


\section{Análisis dieléctrico}

Los resultados de la caracterización dieléctrica de este material se muestran en el diagrama Cole-Cole (figura 3a), el cual presenta un semicírculo centrado bajo el eje de la permitividad real a alta frecuencia y una línea recta a bajas frecuencias. Este comportamiento es típico de un material dominado por conductividad DC donde el proceso de transporte de carga tiene similar constante de tiempo, como se reporta en la literatura. ${ }^{6}$ La especies interlaminares son móviles y contribuyen a este tipo de conductividad debido al efecto cooperativo causado por la polarizabilidad orientacional del anión nitrato.
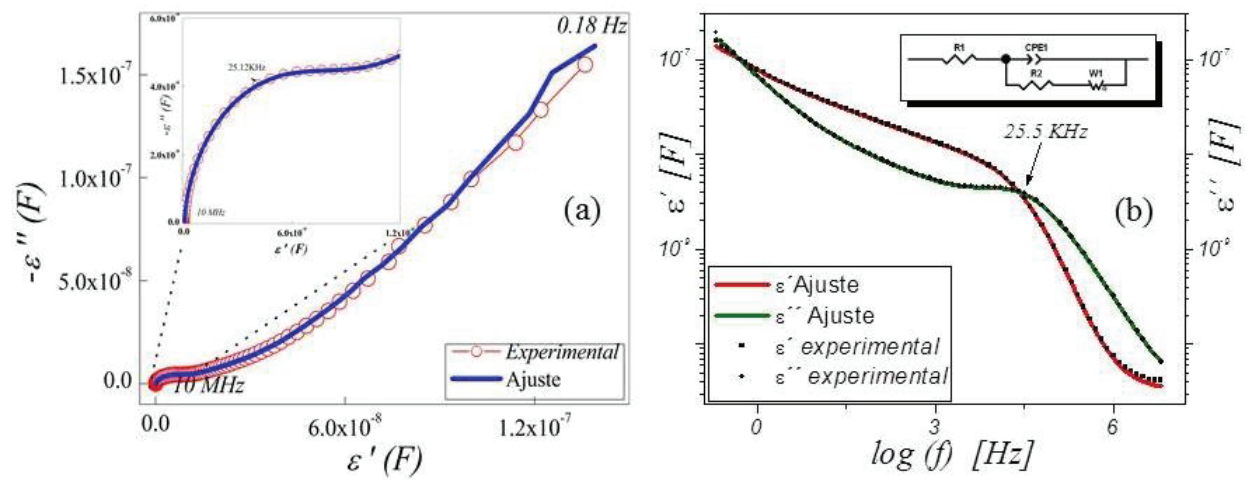

Figura 3. (a) Diagrama de Cole-Cole, (b) Diagrama de Bode para LDH, Inserto circuito equivalente. En ambos diagramas se presenta los resultados del ajuste de los datos experimentales mediante el circuito equivalente.

La dinámica del ion nitrato en el sólido está influida por la distribución de los tiempos de relajación debida a las heterogeneidades relacionadas con la presencia de poros y con áreas de contacto irregulares entre agregados de partículas..$^{13}$ Los dipolos en este material no están localizados en el mismo ambiente químico debido a la presencia de cationes $\mathrm{Mg}$ y $\mathrm{Al}$ en la lámina; de esta manera, algunos dipolos tendrán más libertad de rotar que los otros. ${ }^{14}$ Asimismo, la porosidad y el tamaño de cristal influyen en el comportamiento eléctrico de este tipo de materiales. Es probable que el primer parámetro (porosidad) proporcione el espacio necesario para que el anión nitrato se movilice; así se generan regiones de carga espacial entre las partículas de LDH, en donde es probable que se favorezca la conductividad. ${ }^{15 a} \mathrm{El}$ segundo parámetro (tamaño de cristal), relacionado también con los defectos de apilamiento de las láminas, incrementa la movilidad de los iones debido a que proporciona el ambiente microestructural necesario para las rotaciones de los iones.

El diagrama de Bode de la permitividad real (figura 3b), decrece a medida que la frecuencia se incrementa y presenta un proceso simple de relajación representado por un pico en el diagrama de las pérdidas dieléctricas o permitividad imaginaria $\left(\varepsilon^{\prime \prime}=4461 \mathrm{nF}\right)$ a una frecuencia característica de $25,519 \mathrm{KHz}$ asociado con la rotación de las moléculas de agua en el material. ${ }^{11}$ El valor estimado de la permitividad a esta frecuencia fue de $4105 \mathrm{nF}$. El proceso de relajación del agua no confinada ocurre a $20 \mathrm{GHz}$ con un tiempo de relajación 
de $9,3 \times 10^{-12} \mathrm{~S}$ a $20^{\circ} \mathrm{C},{ }^{15 b}$ mientras que el valor encontrado en el presente trabajo fue $6,34 \times 10^{-}$ ${ }^{6} \mathrm{~S}$, sugiriendo en que ambiente confinado las fuerzas electrostáticas interaccionan con las láminas positivas del $\mathrm{LDH}$, favoreciendo la rotación de los dipolos de las moléculas de agua a una menor frecuencia. Por otro lado, el valor estimado de la constante dieléctrica del LDH fue de 25,30 tomado en un rango de $1 \times 10^{7}$ a 3,1 $1 \times 10^{6} \mathrm{~Hz}$, donde la permitividad real es aproximadamente constante. El circuito equivalente que tiene significado físico y representa los resultados experimentales, se muestran en el inserto de la figura 3b. Este circuito tipo Randles modificado, presenta un resistor (R1) conectado en serie con un elemento de fase constante (CPE1), que a su vez se encuentra en paralelo con un resistor (R2) y un elemento de tipo Warburg (W1) para simular una difusión semi-infinita, en el caso de los portadores de carga iónicos (tabla 1).

R1 representa la resistencia de contacto entre la muestra y el electrodo. La conexión en paralelo de R2 y CPE1 representa el fenómeno resistivo y capacitivo dentro de los poros del material así como el efecto cooperativo de la orientación de los dipolos de las moléculas de agua localizadas en la región interlaminar. Finalmente, W1 representa la difusión del anión nitrato dentro de los poros del material.

Tabla 1. Resultados del ajuste del circuito equivalente

\begin{tabular}{|c|c|c|}
\hline Elemento & Valor & Descripción \\
\hline R1 & $250 \Omega$ & $\begin{array}{l}\text { Resistencia entre el electrodo y } \\
\text { la muestra. }\end{array}$ \\
\hline CPE1-T & $9,1592 \times 10^{-8} \mathrm{~F}$ & \multirow[t]{2}{*}{$\begin{array}{l}\text { Elemento de Fase Constante } \\
\text { que describe el fenómeno } \\
\text { capacitivo dentro de los poros } \\
\text { del material. }\end{array}$} \\
\hline CPE1-P & 0,8 & \\
\hline $\mathrm{R} 2$ & $250 \Omega$ & $\begin{array}{l}\text { Elemento resistivo que } \\
\text { describe el flujo de portadores } \\
\text { de carga dentro del LDH. }\end{array}$ \\
\hline W1-R & $4,5 \times 10^{7} \Omega$ & \multirow{3}{*}{$\begin{array}{lcc}\text { Elemento de tipo Warburg que } \\
\text { describe la difusión } & \text { iónica } \\
\text { dentro de la } & \text { región } \\
\text { interlaminar. } & & \\
\end{array}$} \\
\hline W1-T & $3,5 \times 10^{6} \mathrm{~s}$ & \\
\hline W1-P & 0,14 & \\
\hline
\end{tabular}

CPE (Elemento de Fase Constante cuya ecuación se describe como: $Z=1$ / $[\mathrm{T}(\mathrm{I} * \omega) \mathrm{P}]$

Ecuación de impedancia para el elemento de tipo Warburg abierto: $\mathrm{Z}=\mathrm{R} *\left(\operatorname{ctgh}\left[\mathrm{I}^{*} \mathrm{~T} * \omega\right] \mathrm{P}\right) /(\mathrm{I} * \mathrm{~T} * \omega) \mathrm{P}$.

$\mathrm{W} 1-\mathrm{T}=\mathrm{L} 2 / \mathrm{D}$ (L es el espesor efectivo de difusión y D corresponde al coeficiente efectivo de difusión de la partícula)

En ambas ecuaciones I es la raíz cuadrada de (-1) y w= es la frecuencia angular de la señal AC. 


\section{CONCLUSIONES}

Los resultados de XRD muestran que el LDH de $\mathrm{Mg}_{2} \mathrm{Al}-\mathrm{NO}_{3}$ tiene una estructura laminar con un tamaño estimado de cristal de 5,0 nm y el anión nitrato en la región interlaminar como se evidenció por FT-IR. Los resultados sugieren que hay un ambiente químico favorable para la difusión del ion nitrato que contribuye en gran medida a la conductividad DC a bajas frecuencias. El tiempo de relajación estimado para el agua confinada en el LDH $\left(6,34 \times 10^{-6}\right.$ s) fue menor que la del agua libre sugiriendo que existe un efecto cooperativo con los $\mathrm{OH}^{-}$ localizados en la lámina del material LDH.

\section{AGRADECIMIENTO}

Este trabajo fue financiado por la Dirección de Investigación y Extensión DIMA (Proyecto Cod. 28739) y la Facultad de Ciencias Exactas y Naturales de la Universidad Nacional de Colombia, Sede Manizales. Los autores también agradecen a la Universidad de Alicante, España, por las mediciones de microscopía electrónica de transmisión.

\section{BIBLIOGRAFÍA}

1. Cavani F, Trifirò F, Vaccari A. Hydrotalcite-type anionic clays: Preparation, properties and applications. Catal. Today. 1991;11(2):173-301.

2. $\mathrm{Gu} \mathrm{Z}$, Atherton JJ, Xu ZP. Hierarchical layered double hydroxide nanocomposites: structure, synthesis and applications. Chem. Commun. 2015;51(15):3024-36.

3. Villegas JC, Giraldo OH, Laubernds K, Suib SL. New Layered Double Hydroxides Containing Intercalated Manganese Oxide Species: Synthesis and Characterization. Inorg. Chem. 2003;42(18):5621-31.

4. Han J, Wei M, Evans DG, Duan X. Hierarchical Layered Double Hydroxide Materials. Advanced Hierarchical Nanostructured Materials: Wiley-VCH Verlag GmbH \& Co. KGaA; 2014. p. 231-66.

5. Wang Q, O'Hare D. Recent Advances in the Synthesis and Application of Layered Double Hydroxide (LDH) Nanosheets. Chem. Rev. 2012;112(7):4124-55.

6. Tezuka S, Chitrakar R, Sonoda A, Ooi K, Tomida T. Studies on selective adsorbents for oxo-anions. Nitrate ion-exchange properties of layered double hydroxides with different metal atoms. Green Chem. 2004;6(2):104-9.

7. Rives V. Layered Double Hydroxides: Present and Future. New York: Nova Science Publisher; 2006.

8. Roto R, Villemure G. Electrochemical impedance spectroscopy of electrodes modified with thin films of $\mathrm{Ni}-\mathrm{Al}-\mathrm{Cl}$ layered double hydroxides. J. Electroanal. Chem. 2002;527(1-2):123-30. 
9. Roto R, Villemure G. Electrochemical impedance spectroscopy of electrodes modified with thin films of $\mathrm{MgMnCO} 3$ layered double hydroxides. Electrochim. Acta. 2006;51(12):2539-46.

10. Bugris V, Haspel H, Kukovecz Á, Kónya Z, Sipiczki M, Sipos P, et al. Water Types and Their Relaxation Behavior in Partially Rehydrated CaFe-Mixed Binary Oxide Obtained from CaFe-Layered Double Hydroxide in the 155-298 K Temperature Range. Langmuir. 2013;29(43):13315-21.

11. Frunza L, Schönhals A, Frunza S, Parvulescu VI, Cojocaru B, Carriazo D, et al. Rotational Fluctuations of Water Confined to Layered Oxide Materials: Nonmonotonous Temperature Dependence of Relaxation Times. J. Phys. Chem. A. 2007;111(24):516675.

12 (a) Guo X, Zhang F, Xu S, Cui Z, Evans DG, Duan X. Effects of Varying the Preparation Conditions on the Dielectric Constant of Mixed Metal Oxide Films Derived from Layered Double Hydroxide Precursor Films. Ind. Eng. Chem. Res. 2009;48(24):10864-9. (b) Ivanov M, Klemkaite K, Khinsky A, Kareiva A, Banys J. Dielectric and Conductive Properties of Hydrotalcite. Ferroelectrics. 2011;417(1):136-42.

13. Kao KC. Dielectric Phenomena in solids: with Emphasis on Physical Concepts of Electronic Processes;: Elsevier; 2004.

14. Arias NP, Dávila MT, Giraldo O. Electrical behavior of an octahedral layered OL-1-type manganese oxide material. Ionics. 2013;19(2):201-14.

15. (a) Philippe Knauth; Solid State Ionics. 2006;177: 2495-2502 (b) Raju GG. Dielectrics in Electric Fileds;: Marcel Dekker, Inc. 2003. 